九州大学学術情報リポジトリ

Kyushu University Institutional Repository

Adipose tissue complement factor B promotes adipocyte maturation

松永，紘明

https://doi.org/10.15017/1931833

出版情報：九州大学，2017，博士（歯学），課程博士

バージョン：

権利関係 : ๑ 2017 Elsevier Inc. All rights reserved. 


\title{
Adipose tissue complement factor B promotes adipocyte maturation
}

\author{
Hiroaki Matsunaga a , Misaki Iwashita a, Takanori Shinjo a, Akiko Yamashita a, \\ Mitsudai Tsuruta ${ }^{\mathrm{a}}$, Shoichiro Nagasaka ${ }^{\mathrm{b}}$, Ataru Taniguchi ${ }^{\mathrm{c}}$, Mitsuo Fukushima ${ }^{\mathrm{d}}$, \\ Naoya Watanabe ${ }^{\mathrm{e}}$, Fusanori Nishimura ${ }^{\mathrm{a}, *}$ \\ a Section of Periodontology, Kyushu University Faculty of Dental Science, Fukuoka, Japan \\ b Division of Diabetes, Metabolism and Endocrinology, Showa University Fujigaoka Hospital, Yokohama, Japan \\ ${ }^{\mathrm{c}}$ Division of Diabetes and Endocrinology, Kyoto Preventive Medical Center, Kyoto, Japan \\ ${ }^{\mathrm{d}}$ Preemptive Medicine and Lifestyle-related Disease Research Center, Kyoto University Hospital, Kyoto, Japan \\ ${ }^{\mathrm{e}}$ Health Care and Promotion Center, Yodogawa Christian Hospital, Osaka, Japan
}

\section{A R T I C L E I N F O}

\section{Article history:}

Received 7 November 2017

Accepted 10 November 2017

Available online $\mathrm{xxx}$

\section{Keywords:}

Complement factor B

Adipocyte maturation

Obesity

\begin{abstract}
A B S T R A C T
Objectives: It is well-known that the complement system plays an essential role in host immunity. Observational studies have indicated that complement system-related molecules such as complement factor B (CfB) and other components are correlated with obesity and/or insulin resistance parameters. In this study, we investigated the role of adipocyte-derived $\mathrm{CfB}$ in adipose tissue metabolism.

Methods: We investigated the expression level of complement system-related genes in adipocytes. To understand the role of $\mathrm{CfB}$ in adipocyte, we performed $\mathrm{Cfb}$ overexpression in 3T3-L1 preadipocytes and generated adipocyte-specific $C f b$ transgenic mice.

Results: $C f b$ expression was markedly enhanced in 3T3-L1 adipocytes co-cultured with macrophages following endotoxin stimulation. In $C f b$-overexpressing cells, the expression of adipocyte differentiation/ maturation-related genes encoding peroxisome proliferator-activated receptor $\gamma$ (Ppar $\gamma$ ), adipocyte Protein 2 and perilipin was significantly enhanced. $C f b$ transgenic mice showed a marked increase in the expression of genes encoding Ppar $\gamma$, perilipin, sterol regulatory element-binding protein $1 \mathrm{c}$, and $\mathrm{Cd} 36$ in the subcutaneous adipose tissue.

Conclusions: CfB plays a crucial role in late-phase of adipocyte differentiation and subsequent lipid droplet formation.
\end{abstract}

(c) 2017 Elsevier Inc. All rights reserved.

\section{Introduction}

It is well-established that adipose tissue inflammation plays a crucial role in inducing insulin resistance in obese subjects [1,2]. However, recent reports have suggested that changes in gut microflora play an important additional role in the pathogenesis of obesity, cardiovascular disease, and diabetes by accelerating adipose tissue inflammation [3], since such changes may be associated with decreased epithelial integrity and with increased concentrations of toll-like receptor 4 (TLR4) ligands such as bacterial endotoxin and lipopolysaccharide (LPS) in the portal vein [4]. We hypothesized that LPS in the portal vein would activate circulating monocyte, and that these activated monocytic cells would migrate

\footnotetext{
* Corresponding author. Section of Periodontology, Kyushu University Faculty of Dental Science, 3-1-1 Maidashi, Higashi-ku, Fukuoka 812-8582, Japan.

E-mail address: fusanori@dent.kyushu-u.ac.jp (F. Nishimura).
}

into adipose tissue to differentiate into macrophages and interact with adipocytes, further upregulating adipose tissue inflammation. Based on this hypothesis, we compared the gene expression profile in adipocytes co-cultured with macrophages in the presence or absence of bacterial LPS, and observed marked upregulation of the genes encoding LPS-binding proteins and CD14, downregulation of glucose transporter 4, and upregulation of suppressor of cytokine signaling 3, indicating acceleration of inflammation and exacerbation of the insulin resistant state [5]. Among the genes where expression was dramatically enhanced by LPS stimulation, complement factor $\mathrm{B}(C f B)$ gene expression was markedly upregulated. It is well known that the complement system plays an important role in host immunity through the recognition and elimination of infectious foreign bodies. There are three major pathways in the complement system [6]. Among these three, the second or alternative pathway is activated by the binding of complement component 3 (C3) to pathogenic bacteria. C3 is the most 
abundantly observed complement system associated molecule, and $\mathrm{CfB}$ is essential for the activation of the second pathway [7]. Interestingly, serum C3 and/or complement component 4 (C4) concentration or adipose tissue $C f B$ gene expression are reported to be associated with the incidence rate of type 2 diabetes and other metabolic diseases [8-11]. All these observational studies strongly indicate that the complement system may play an important role in the pathogenesis of obesity and/or diabetes, although the precise molecular basis is not well understood yet. Therefore, in this study, we were interested in observing the effects of $\mathrm{CfB}$ overexpression at both the cellular and systemic level to understand the role of CfB in adipose tissue metabolism.

\section{Materials and methods}

\subsection{Cells and cell culture}

Murine 3T3-L1 preadipocytes [American Type Culture Collection (ATCC), Manassas, VA, USA] were maintained in Dulbecco's modified Eagles medium (DMEM) (Nacalai Tesque, Kyoto, Japan) containing $10 \%$ calf serum in an atmosphere of $10 \% \mathrm{CO}_{2}$ at $37{ }^{\circ} \mathrm{C}$. Two days after the 3T3-L1 fibroblasts had reached confluence, differentiation was induced by treating the cells with DMEM containing $4 \mu \mathrm{g} / \mathrm{ml}$ dexamethasone (Sigma Aldrich, St. Louis, MO, USA), 0.5 mM 3-isobutyl-1-methylxanthine (Sigma Aldrich), 200 nM insulin (Cell Science \& Technology Institute, Inc., Sendai, Japan) and $10 \%$ fetal bovine serum (FBS) (Biowest, Nuaillé, France) for $48 \mathrm{~h}$, as described previously [12]. Cells were fed with DMEM containing $10 \%$ FBS and $1 \mu \mathrm{M}$ insulin every other day. Co-culture of adipocytes and macrophages was performed using a transwell system (Corning Inc., Acton, MA, USA) with a $0.4 \mu \mathrm{m}$ porous membrane to separate the upper and lower chambers. $5 \times 10^{4}$ differentiated 3T3L1 cells were cultured in the lower chamber, while $1 \times 10^{5}$ RAW264.7 cells (ATCC) were cultured in the upper chamber [13].

\subsection{Animals}

$\mathrm{Tg}$ founder mice (F0) expressing the $C f b$ gene specifically in adipose tissue ( $C f b \mathrm{Tg}$ mice) were generated using the adipocyte specific promoter, adipocyte Protein 2 (aP2) [14] from TransGenic Inc. (Fukuoka, Japan) as shown in Supplemental Fig. 1. Male Cfb Tg mice and their male wild type (WT) littermates were fed a normal diet (CRF-1, Oriental Yeast Co. Ltd., Tokyo, Japan) from the age of 4 weeks. Food intake was recorded by subtracting the amounts of food left in the cage from the daily amounts given. At 20 weeks of age, the mice were sacrificed and tissue samples were collected. Male C57BL/6J mice (Jackson Laboratory, Bar Harbor, ME, USA) were provided a normal diet or a high fat diet (60\% of calories from fat, HFD-60, Oriental Yeast Co. Ltd.) for 16 weeks. At 23-25 weeks of age, mice were sacrificed and blood samples were collected. All mice were housed under climate-controlled conditions with a $12 \mathrm{~h}$ light/dark cycle, and with food and water ad libitum. These protocols were approved by the institutional review board of Kyushu University.

\subsection{Analysis of human serum}

We measured serum CfB levels in 374 subjects who underwent health examinations at Yodogawa Christian Hospital. The ethics committee of Yodogawa Christian Hospital approved the protocol and informed consent was obtained from every participant. All subjects had ingested at least $150 \mathrm{~g}$ of carbohydrates for 3 consecutive days prior to the study. Blood was drawn from the antecubital vein, the morning after a $12 \mathrm{~h}$ fast.

\subsection{Oil red $O$ staining}

Cells were washed in PBS, fixed with $10 \%$ buffered formalin for $60 \mathrm{~min}$ at $4{ }^{\circ} \mathrm{C}$ and stained for $30 \mathrm{~min}$ with $0.5 \%$ Oil Red $\mathrm{O}$ solution (Sigma Aldrich). Stained cells were photographed and droplet size was determined using a microscope (BZ-9000 BZ-X Analyzer, Keyence, Osaka, Japan). Plates were washed and treated with isopropanol and the lipid accumulation was quantified by measuring absorbance at an optical density of $540 \mathrm{~nm}$ with a microplate reader (Thermo Scientific ${ }^{\mathrm{TM}}$ Multiskan ${ }^{\mathrm{TM}} \mathrm{FC}$, Thermo, Tokyo, Japan).

\subsection{Enzyme linked immunosorbent assay (ELISA)}

Serum CfB and insulin levels were measured using ELISA kits (Cloud-Clone Corp, WUH, USA and Institute of Biological Sciences, Inc., Kanagawa, Japan), according to the manufacturer's instructions.

\subsection{Transfection}

One day prior to transfection, 3T3-L1 cells were plated to achieve $80-90 \%$ confluence at the time of transfection. Cells were transfected with pCMV (OriGene, MD, USA) or pCMV-Cfb (MC200474, OriGene) plasmid using FuGENE ${ }^{\circledR}$ Transfection Reagent (Promega, WI, USA), according to the manufacturer's instructions. Two days after the transfection, cells were treated with differentiation medium, as described above. Total RNA was extracted from the 3T3-L1 cells every 2 days during induction of adipocyte differentiation.

\subsection{Histological analysis}

Tissue samples were fixed in $4 \%$ paraformaldehyde solution (Nacalai Tesque). The paraffin-embedded sections were stained with hematoxylin and eosin. Adipocyte sizes were measured in four randomly chosen microscopic areas from three animals per group using a microscope (BZ-9000, Keyence BZ-X Analyzer). Average adipocyte size was calculated by dividing the chosen microscopic area by the total adipocyte number in the area.

\subsection{Real-time polymerase chain reaction (PCR)}

Total RNA was extracted from the cells, and tissues homogenized in TRIzol (Thermo Fisher Scientific) and reverse transcribed using Prime Script RT reagent Kits (Takara, Shiga, Japan). The protocol for the reverse transcription cycle was $42{ }^{\circ} \mathrm{C}$ for $30 \mathrm{~min}$ and $95{ }^{\circ} \mathrm{C}$ for $2 \mathrm{~min}$. RT-PCR was performed using the KAPA SYBR ${ }^{\circledR}$ FAST qPCR Kits (Kapa Biosystems, MA, USA) and Step One Plus Real Time PCR System (Applied Biosystems, CA, USA). PCR was carried out in two steps, the first at $95^{\circ} \mathrm{C}$ for $5 \mathrm{~s}$ and the second at $60{ }^{\circ} \mathrm{C}$ for $30 \mathrm{~s}$, which were then repeated 40 times. Relative mRNA genes were normalized to the GAPDH mRNA level and relative expression levels were determined by the comparative $\mathrm{Ct}$ method. Primer sequences are shown in Supplemental Table 1.

\subsection{Western blot analysis}

The cells and tissue sections were solubilized with CytoBuster ${ }^{\mathrm{TM}}$ Protein Extraction Reagent (Millipore, Billerica, MA, USA). Equal amounts of protein from whole cell lysates were resolved by SDSPAGE. The proteins were then transferred to polyvinylidene difluoride membranes (Millipore) using the semi-dry system [Trans-Blot SD Semi-Dry Transfer Cell (Bio-Rad Laboratories, Hercules, CA, USA)]. Primary antibodies were as follows: anti-mouse $\beta$ actin (Abcam, Cambridge, UK) and anti-mouse CfB (Sigma Aldrich). 
After incubation with secondary antibodies (HRP-conjugated antirabbit and anti-mouse, Cell Signaling Technology, Inc.), immunoreactive proteins were visualized using enhanced chemiluminescence (Chemi-Lumi One Super, Nacalai Tesque), and signals were analyzed using Image Quant LAS4000 (GE Healthcare, Chalfont, UK).

\subsection{Glucose tolerance test (GTT) and insulin tolerance test (ITT)}

Mice were injected with D (+)-glucose ( $2 \mathrm{~g} / \mathrm{kg}$ body weight) or insulin ( 0.75 unit $/ \mathrm{kg}$ body weight) intraperitoneally after $6 \mathrm{~h}$ of fasting. Blood samples were collected from the tail vein at $0,15,30$, 60,90 and 120 min after the injection. Blood glucose concentrations were determined with a glucose sensor (Medisafe mini, Terumo, Tokyo, Japan).

\subsection{Statistical analysis}

Data are presented as mean \pm SD. Statistical comparisons of data from two groups were performed using a Student's $t$-test. Associations among insulin resistance indexes and serum CfB levels were evaluated by Spearman's correlation coefficient using SPSS/w17.0 (SPSS, Chicago, IL, USA). Values of $P<0.05$ were considered statistically significant.

\section{Results}

\subsection{CfB protein is abundantly expressed in inflamed adipose tissue and serum $C F B$ concentration increases in obesity}

We first tried to confirm the high expression of $C f b$ gene in 3T3L1 differentiated adipocytes co-cultured with RAW 264.7 cells by LPS stimulation. As shown in Fig. $1 \mathrm{~A}, \mathrm{Cfb}$ expression was markedly increased following LPS stimulation, which was consistent with our previous microarray data. Conversely, $C f d, C f h$ and $C 3$ gene expression was not influenced by LPS stimulation (Fig. 1A). We then measured serum $\mathrm{CfB}$ concentration in 374 subjects undergoing a regular medical check-up and observed its association with other biochemical and inflammatory parameters. As this was a regular medical check-up, subjects with acute illnesses were not included. The profiles of all subjects are shown in Fig. 1B. Serum CfB concentration was positively associated with BMI, homeostasis model assessment insulin resistance index (HOMA-IR), insulin, and tumor necrosis factor- $\alpha$ (TNF- $\alpha$ ) levels (Fig. 1C). Furthermore, the mice fed a high fat diet exhibited a higher concentration of CfB levels than mice fed a normal diet (Fig. 1D). These data indicate that serum CfB concentration increases in obesity and may play a role in its pathogenesis.

\subsection{Overexpression of $C f b$ in preadipocytes promotes adipocyte differentiation, maturation and lipid accumulation}

To see the role of $\mathrm{CfB}$ on adipocyte function and differentiation, we transfected adipocytes with $C f b$ (pCMV- $C f b$ ) or vector alone (pCMV). In $C f b$ overexpressing cells, expression of genes encoding peroxisome proliferator-activated receptor $\gamma$ (Ppar $\gamma$ ), CCAATenhancer-binding proteins $\alpha(\mathrm{C} / \mathrm{ebp} \alpha), \mathrm{C} / \operatorname{ebp} \beta, \mathrm{aP} 2$, leptin and perilipin was significantly enhanced at the late stage of differentiation (Fig. $2 \mathrm{~A}$ and $\mathrm{B}$ ), suggesting that $\mathrm{CfB}$ promotes adipocyte differentiation and maturation. Oil red $\mathrm{O}$ staining indicated that the overexpression of $\mathrm{CfB}$ caused a significant enlargement in cell size and lipid accumulation (Fig. 2C-E). In Cfb-overexpressing 3T3-L1 cells, at 10 days after the induction of differentiation, expression of the genes encoding acetyl-CoA carboxylase (Acc), which is associated with fatty acid synthesis, $\mathrm{Cd} 36$, associated with fatty acid intake, and sterol regulatory element-binding protein $1 \mathrm{c}$ gene (Srebp1c), associated with lipid synthesis, were all up-regulated, while the expression of acyl-CoA oxidase I (Acox1) gene, associated with fatty acid oxidation, was down-regulated (Fig. 2F). From these results, it was suggested that $\mathrm{CfB}$ further enhanced gene expression, which is usually up-regulated at mid-to late-adipogenic stages, and influenced lipid metabolism.

\subsection{Adipocyte-specific Cfb overexpressing mice showed increased body weight and impaired insulin sensitivity}

To understand the in vivo effects of adipocyte-derived $\mathrm{CfB}$, we generated adipocyte-specific $C f b$ overexpressing mice (Fig. $3 \mathrm{~A}$ and B and Supplemental Fig. 1). A normal diet was fed to both $C f b \mathrm{Tg}$ and WT control mice from 4 weeks of age. Although food intake did not differ significantly between the two groups, body weight in $\mathrm{Cfb} \mathrm{Tg}$ mice was kept higher after 8 weeks of age than control mice (Fig. $3 \mathrm{C}$ and D). After the difference in body weight became apparent, GTT and ITT were performed at 16 and 18 weeks, respectively. As shown in Fig. 3E, Cfb Tg mice showed a decreasing trend in glucose tolerance 30 min after glucose loading. Moreover, the glucose-lowering effect of insulin was significantly impaired in the $C f b \mathrm{Tg}$ mice compared to that in the WT mice (Fig. 3F). Fasting insulin concentration was slightly higher in $C f b \mathrm{Tg}$ mice, although the difference was not statistically significant (Fig. 3G). At 20 weeks, mice were sacrificed and adipose tissues were isolated. Serum CfB concentration was significantly enhanced in Tg mice compared to WT mice (Fig. $3 \mathrm{H}$ ). Cfb Tg mice exhibited a significantly higher amount of inguinal white adipose tissue (iWAT) and epidermal white adipose tissue (eWAT) than WT mice (Fig. 3I). Similar results were obtained when the data were expressed as a percentage of total body weight (Fig. 3J). Average adipocyte size of iWAT and eWAT was much larger in $C f b \mathrm{Tg}$ mice than in WT control mice (Fig. 3J and $\mathrm{K}$ ). In contrast, the tissue weight and cell size of interscapular brown adipose tissue (BAT) did not differ significantly between the two groups (Fig. 3I-K).

\subsection{Marked increase in the expression levels of genes associated with adipocyte differentiation and maturation in subcutaneous adipose tissue of $\mathrm{Cfb} \mathrm{Tg}$ mice}

We then measured the expression levels of genes associated with adipocyte differentiation and lipid deposition, glucose and lipid metabolism and inflammation in iWAT, eWAT and BAT for each mouse. In $C f b \mathrm{Tg}$ mice, iWAT monocyte chemoattractant protein-1 (Mcp-1) expression was significantly increased (Fig. 4A). F4/ 80 positive cells were hardly detectable in adipose tissues of normal diet fed Tg and WT mice (data not shown), suggesting that inflammatory macrophage infiltration was very limited. Interestingly, in addition to $C f b$, which activates the complement pathway, $C 3$ and $C f d$ expression was enhanced, while $C f h$ expression, which is known to suppress the alternative pathway, was downregulated (Fig. 4A). These gene expression levels did not differ significantly in the liver, eWAT and iWAT between $C f b \mathrm{Tg}$ mice and WT control mice (data not shown). Expression of genes encoding Ppar $\gamma$, aP2, Krüppel-like factor 5 (Klf5) and leptin expression which are all closely associated with adipocyte differentiation and maturation, and perilipin, which is associated with an increase in lipid droplet and lipolysis, was markedly enhanced in the iWAT of $C f b \mathrm{Tg}$ than in that of WT mice (Fig. 4B). Furthermore, the expression of the Srebp1c, which is associated with lipid synthesis and $C d 36$, which is 
$\mathrm{A}$
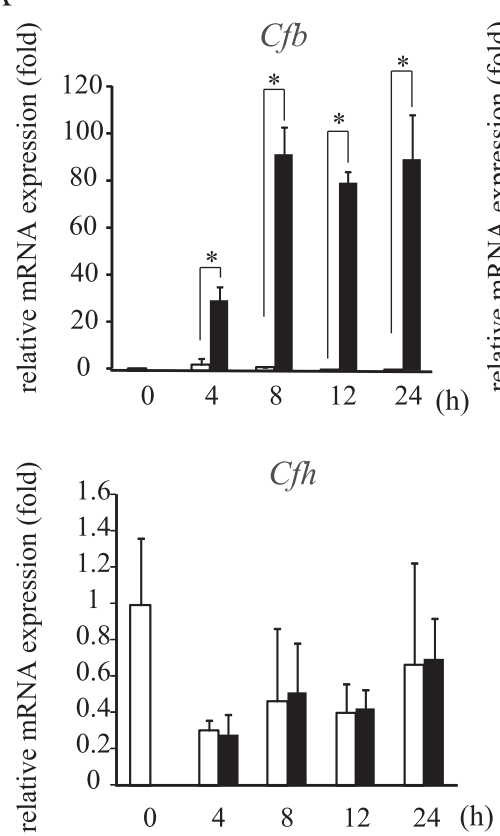

응 $\quad C 3$

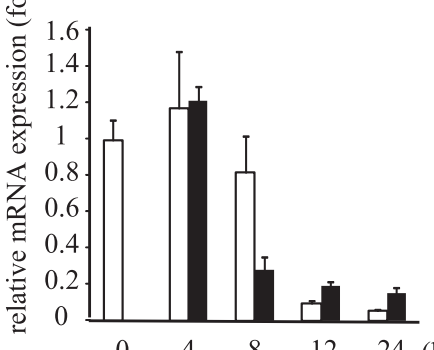

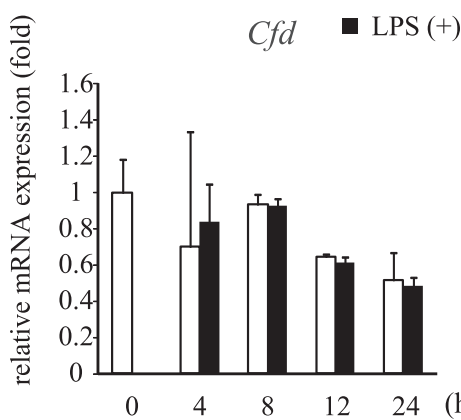

B

\begin{tabular}{lc} 
Characteristics of subjects $(\mathrm{N}=374)$ \\
\hline \hline Age $(\mathrm{yrs})$ & $56.1 \pm 9.35(33-82)$ \\
Sex $(\mathrm{men} /$ women $)$ & $271 / 103$ \\
BMI $\left(\mathrm{kg} / \mathrm{m}^{2}\right)$ & $23.3 \pm 3.05(15.5-33.8)$ \\
CRP $(\mathrm{mg} / \mathrm{dl})$ & $0.12 \pm 0.32(0-4.42)$ \\
insulin $(\mu \mathrm{U} / \mathrm{ml})$ & $4.24 \pm 2.88(0.5-27.6)$ \\
HOMA-IR* & $1.02 \pm 0.71(0.12-5.93)$ \\
TNF- $\alpha(\mathrm{pg} / \mathrm{ml})$ & $1.54 \pm 1.06(0.004-11.4)$ \\
CfB $(\mu \mathrm{g} / \mathrm{ml})$ & $220 \pm 57.4(66.3-446)$ \\
\hline
\end{tabular}

*HOMA-IR was calculated as glucose $(\mathrm{mg} / \mathrm{dl}) \times$ insulin $(\mu \mathrm{U} / \mathrm{ml}) \div 405$

C

CRP

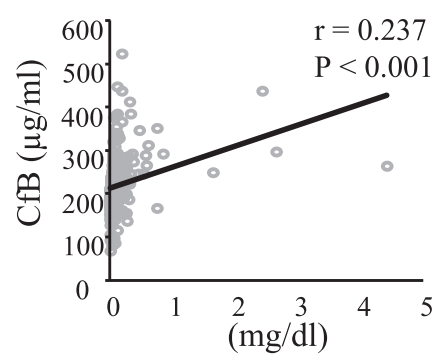

BMI

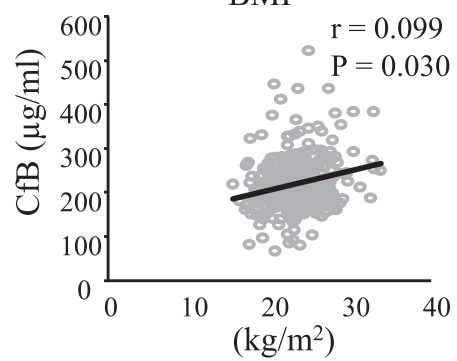

insulin

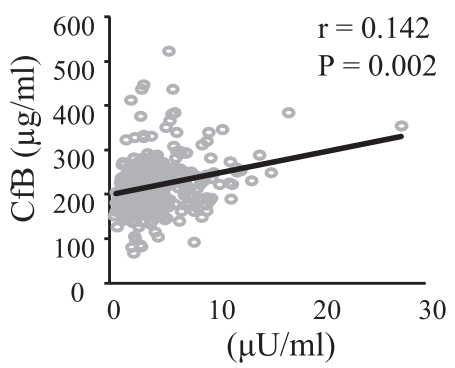

HOMA-IR

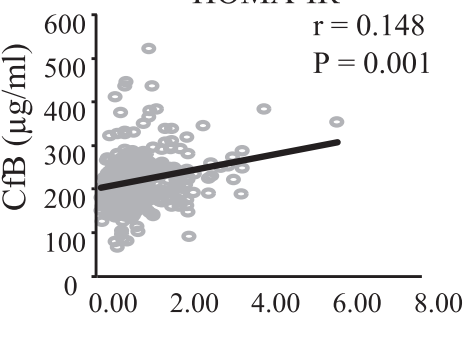

$\mathrm{D}$ body weight

\section{$\mathrm{CfB}$}
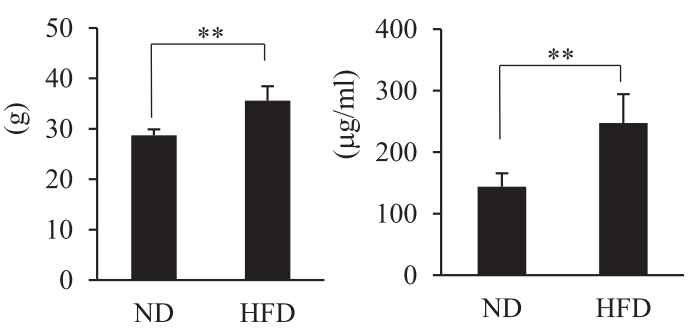

Fig. 1. Complement system gene expression in adipocytes and the association between serum CfB concentration and other biochemical and immunological parameters in Japanese subjects. A) Real time PCR analysis of the genes associated with the complement system in 3T3-L1 differentiated (14 days following differentiation) adipocytes co-cultured with RAW264.7 stimulated with $E$. coli LPS $(1 \mathrm{ng} / \mathrm{ml})$. Representative data from three independent experiments are shown. ${ }^{*} P<0.05 ; \mathrm{n}=3$; Student's $t$-test. B) Subject characteristics. C) Association between serum CfB concentration and several parameters of insulin resistance and inflammatory markers in 374 Japanese subjects who underwent a regular medical check-up. D) Serum CfB concentration at 23-25 weeks in C57BL/6J mice fed either a normal or high fat diet for 16 weeks. ${ }^{* *} P<0.01$; ND, normal diet; HFD, High fat diet; $\mathrm{n}=4$ per group; Student's $t$-test. 
A
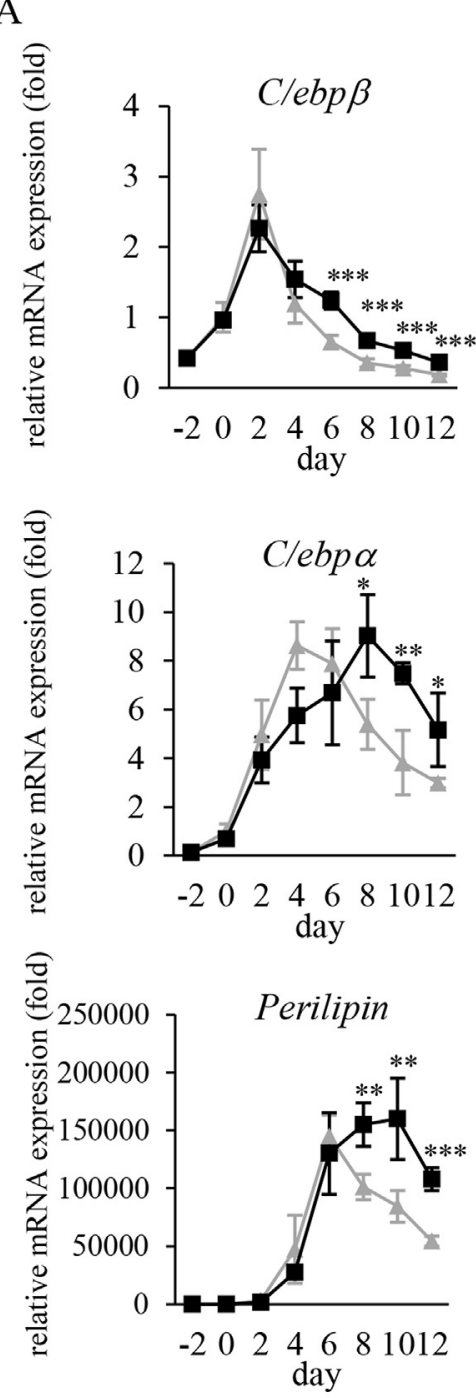
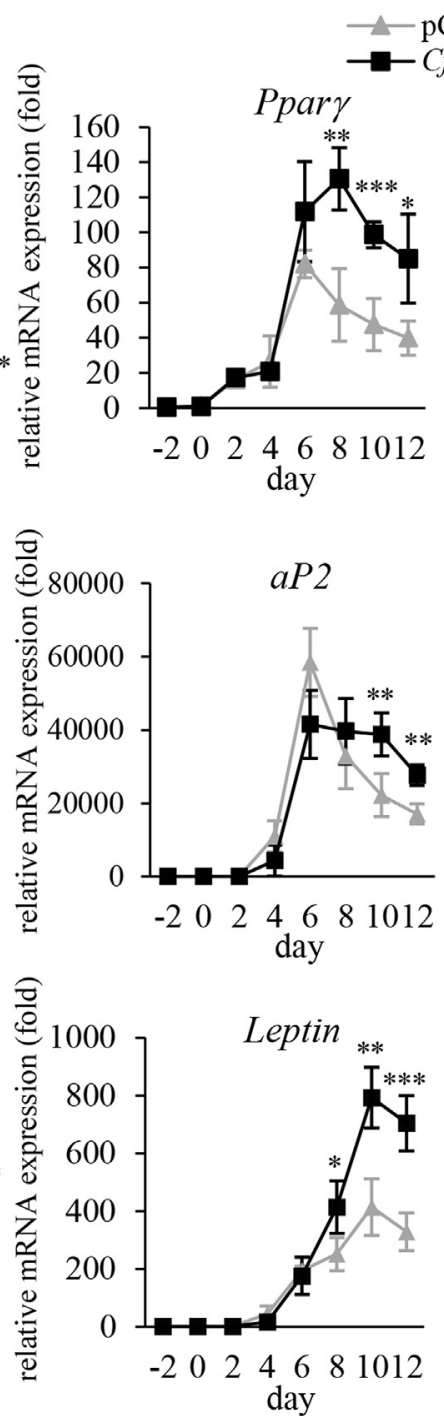

B

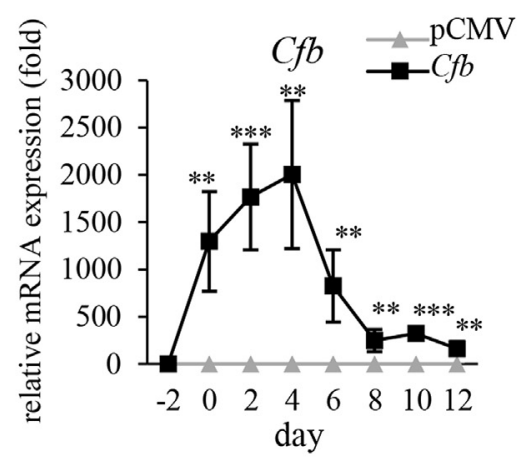

$\mathrm{C}$

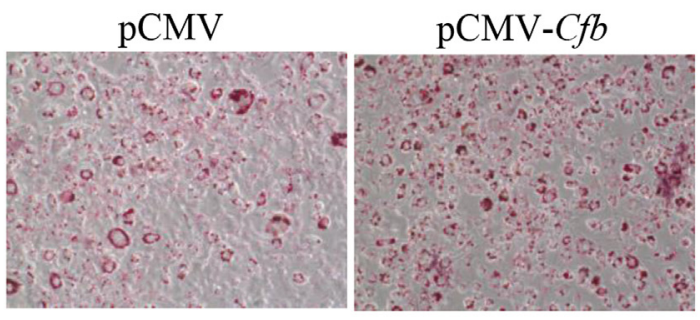

$\mathrm{D}$
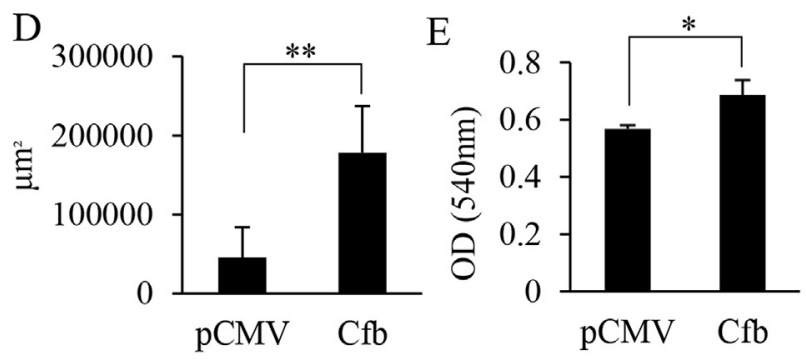

$\mathrm{F}$

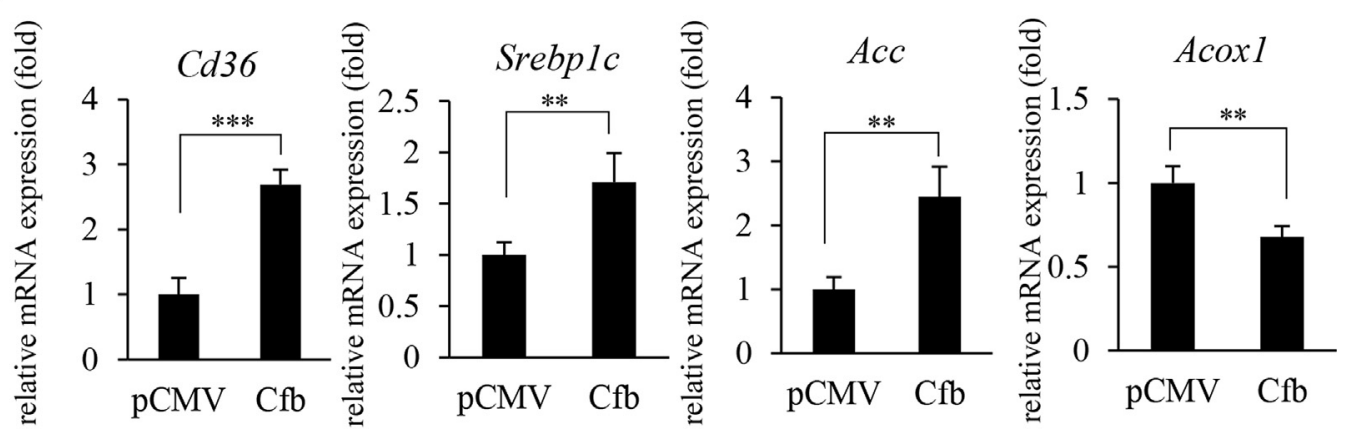

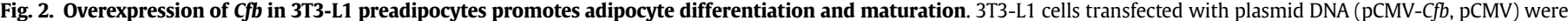

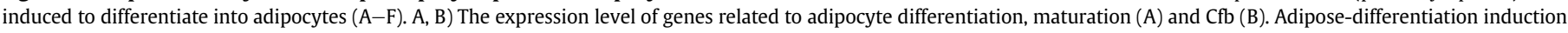

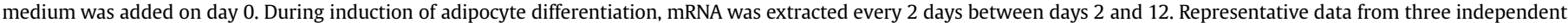

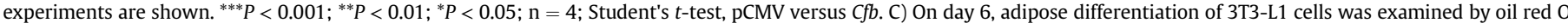

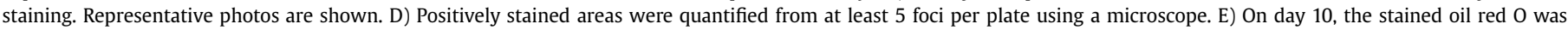

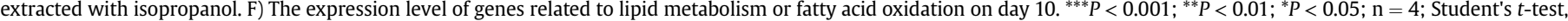
pCMV versus $C f b$.

associated with fatty acid endocytosis, was upregulated in the iWAT of $C f b \mathrm{Tg}$ mice when compared to WT mice (Fig. 4B). The expression of plasminogen activator inhibitor 1 gene (Pai1), which produces a gene product secreted from enlarged adipocytes, was upregulated in the iWAT and eWAT of $C f b$ Tg mice when compared to WT mice
(Fig. 4C). The expression of the gene for Acc was significantly increased in the iWAT, eWAT and BAT of $C f b \mathrm{Tg}$ mice when compared to control mice (Fig. 4D). These results suggest that $\mathrm{Cfb} \mathrm{Tg}$ mice exhibit fat mass expansion. 
A

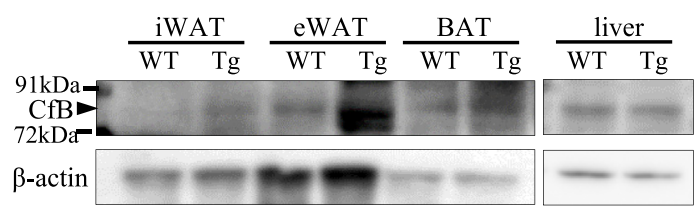

C

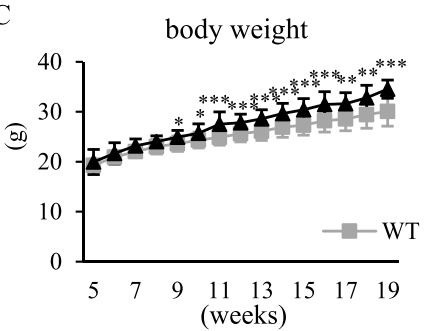

E
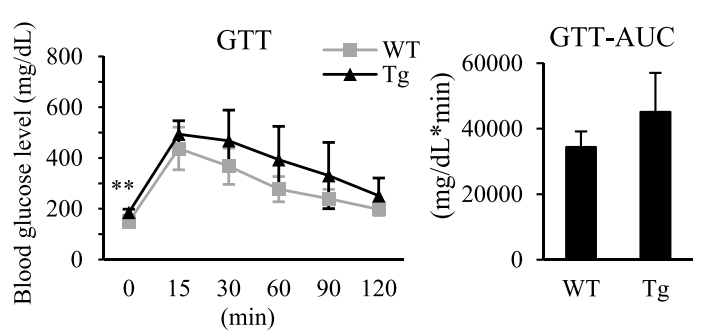

$\mathrm{D}$

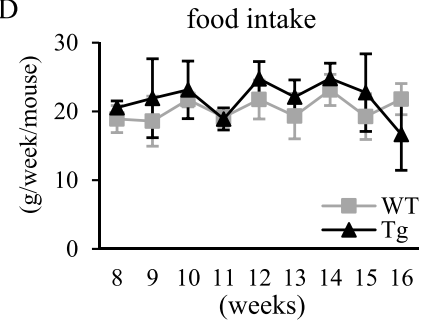

B

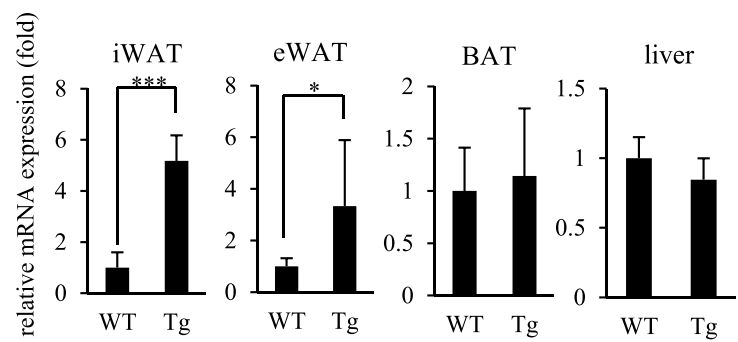

G
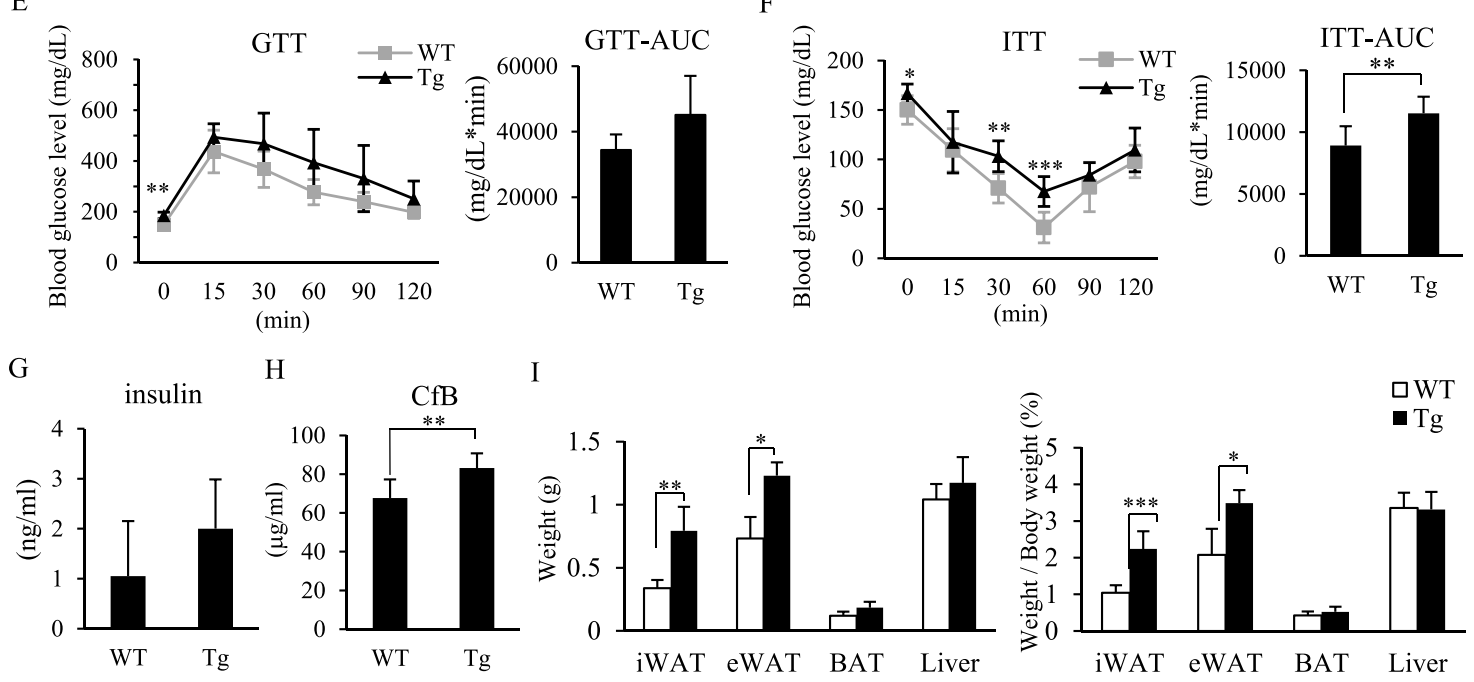

eWAT

BAT
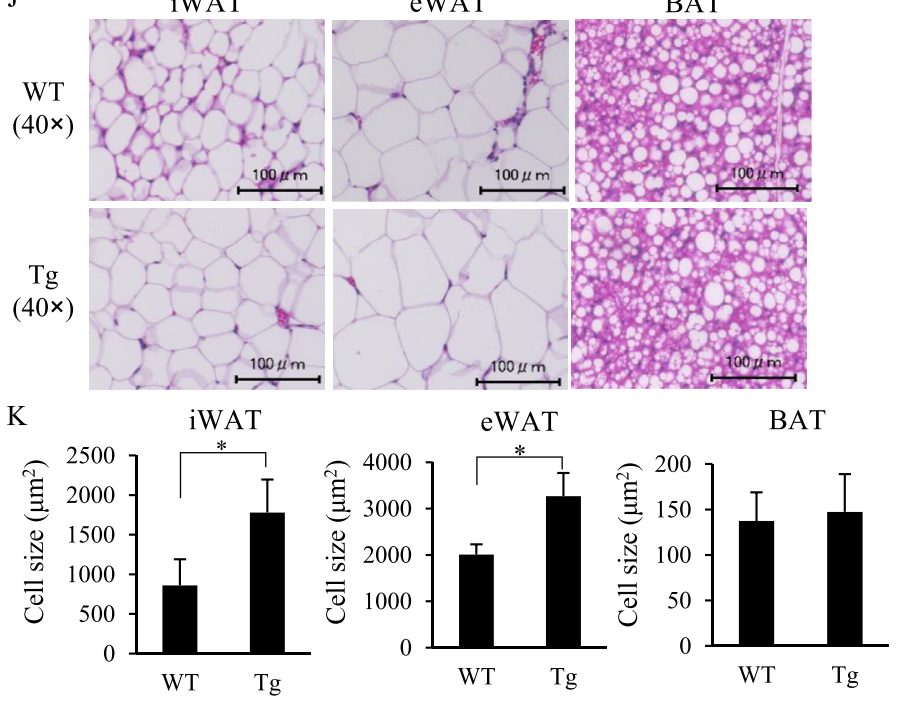

eWAT

BAT

Fig. 3. Characteristics of adipocyte-specific $\boldsymbol{C f b}$ overexpressing mice. A) CfB expression in inguinal white adipose tissue (iWAT), epidermal white adipose tissue (eWAT), interscapular brown adipose tissue (BAT) and liver was determined by western blotting. Size markers are shown at the left and of the image; the arrowhead indicates the size of CfB. B) The expression level of $C f b$ in iWAT, eWAT, BAT and liver. C) Changes in body weight were monitored. D) Mean weekly food intake. E) Mice were injected with glucose intraperitoneally after $6 \mathrm{~h}$ of fasting, at 16 weeks of age. Blood samples were collected from the tail vein at indicated time points. F) Mice were injected with insulin intraperitoneally after $6 \mathrm{~h}$ of fasting, at 18 weeks of age. Blood samples were collected from the tail vein at indicated time points. G) Fasting serum insulin level (20 weeks of age). $\mathrm{H}$ ) Serum concentration of CfB (20 weeks of age). I) Tissue weight (20 weeks of age). J) Representative images of H\&E stained adipose tissue sections. Scale bar, $100 \mu \mathrm{m}$ for $40 \times$ magnification. K) Average adipocyte size. Cell sizes were measured in four randomly chosen microscopic areas from three animals per group. AUC, Area under the curve; ${ }^{* * *} P<0.001 ;{ }^{* *} P<0.01 ;{ }^{*} P<0.05$; WT $(\mathrm{n}=10) ; \operatorname{Tg}(\mathrm{n}=5)$; Student's $t$-test, WT versus Tg. 
A
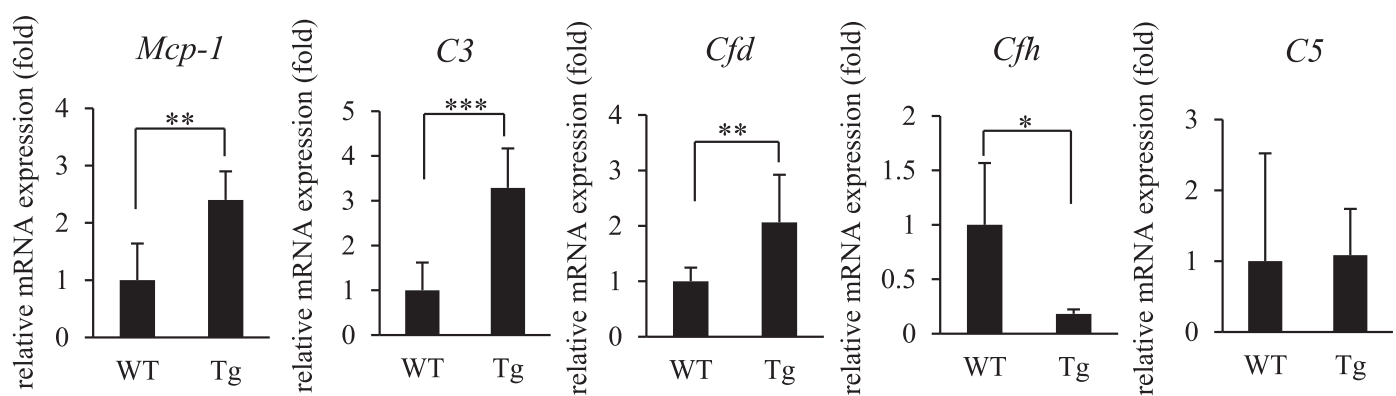

$\mathrm{B}$
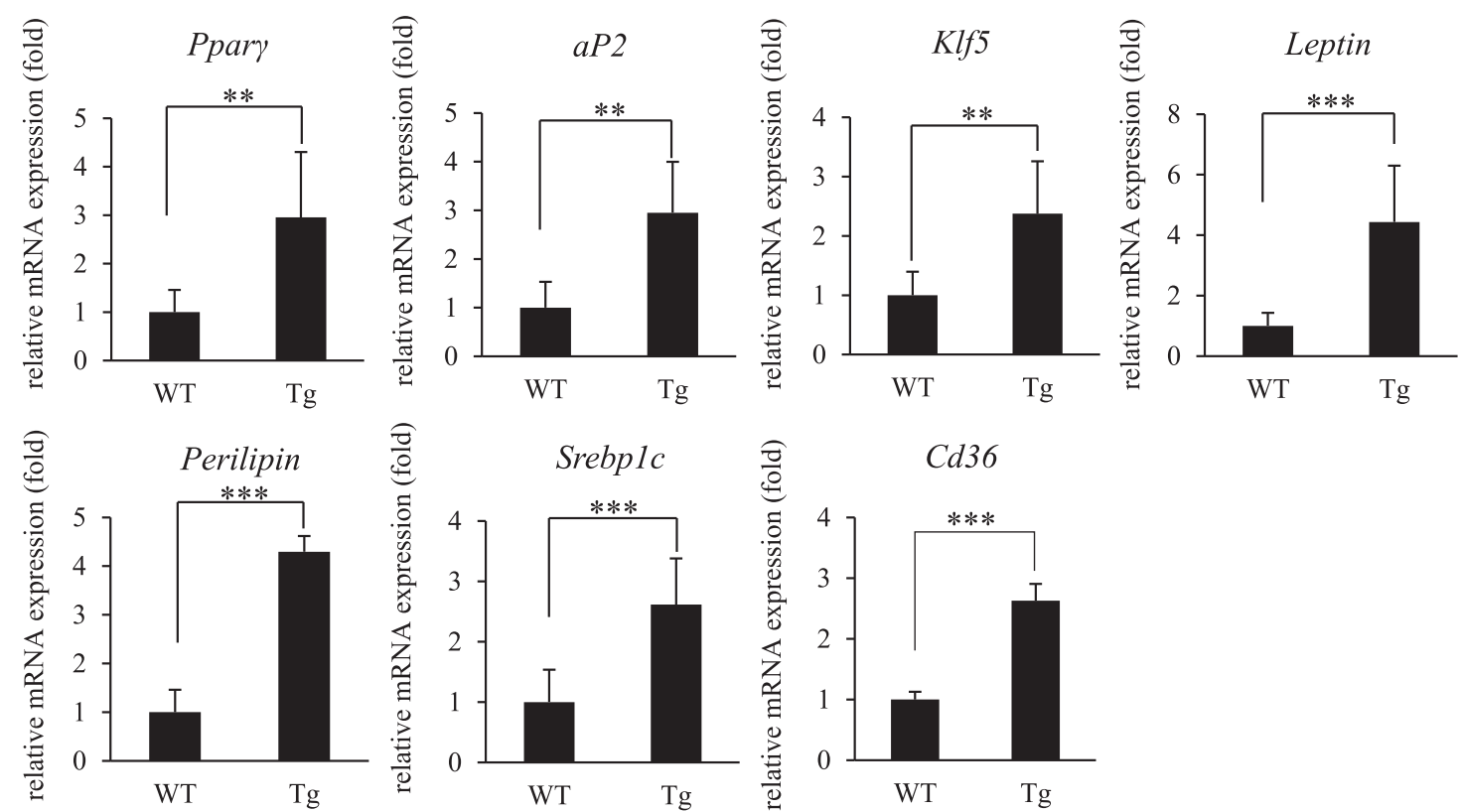

$\mathrm{C}$
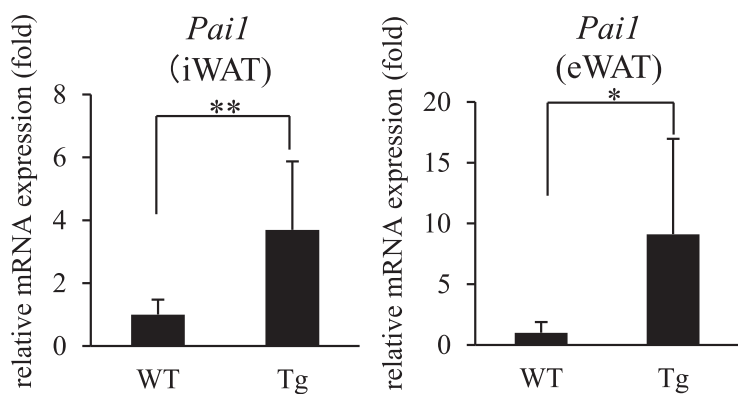

$\mathrm{D}$
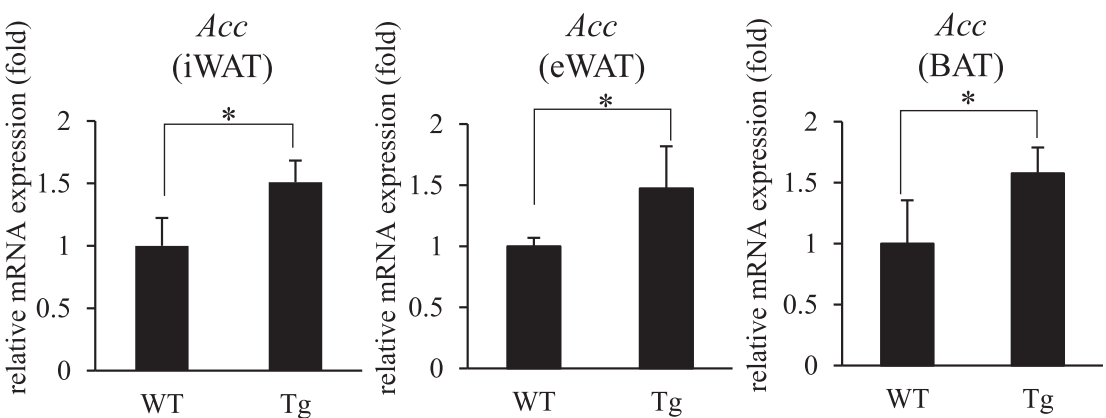

Fig. 4. Effects of Cfb expression on gene expression associated with inflammation, complement system and adipocyte differentiation in iWAT of $C f b$ Tg mice. At 20 weeks of age, the mice were sacrificed and tissue samples were collected. A) The expression level of genes related to inflammation and complement system in iWAT. B) The expression level of genes related to adipocyte differentiation, maturation and lipid accumulation in iWAT. C, D) The expression level of Pai1 and Acc. ${ }^{* * *} P<0.001 ;{ }^{* *} P<0.01 ;{ }^{*} P<0.05 ;$ WT, wild type littermates $(\mathrm{n}=8) ; \mathrm{Tg}, \mathrm{Cfb} \operatorname{Tg}(\mathrm{n}=3)$; Student's $t$-test. 


\section{Discussion}

In this study, we found that $\mathrm{CfB}$ appeared to promote adipocyte differentiation or maturation and subsequent lipid droplet formation. When $\mathrm{CfB}$-overexpressing 3T3-L1 pre-adipocytes were differentiated into mature adipocytes, late-phase adipogenic gene expression of Ppar $\gamma, C / \operatorname{ebp} \alpha$, and Srebp1c, which are associated with lipid synthesis, was markedly up-regulated, and was followed by lipid deposition in the cells. In contrast, we observed only a slight difference in the expression level of $C / \operatorname{ebp} \beta$, which is an early adipogenic gene (Fig. 2A-F). Similar observations have been previously reported, in which early adipogenic gene expression was less influenced by gene overexpression or deletion, while late adipogenic gene expression was more profoundly influenced, resulting in impaired lipid deposition $[15,16]$. Therefore, we speculated that CfB up-regulated Ppar $\gamma$ expression, which drives SREBP1c, ACC, and CD36 expression, resulting in adipocyte maturation and lipid deposition. Transcriptional regulation by PPAR $\gamma$ plays a major role in adipocyte differentiation. In $C f b \mathrm{Tg}$ mice, we observed a marked increase in the expression of genes encoding Ppar $\gamma$, perilipin, Srebp1c and Cd36 in iWAT compared to WT mice. Perilipin is an adipocyte-specific protein induced by PPAR $\gamma$ [17] that surrounds lipid droplets to regulate their size, triglyceride storage and lipolysis [18]. It also has been shown that SREBP-1 transcription factor activation is impaired in perilipin knock out mice, which is known to promote the expression of several genes associated with fatty acid and triglyceride synthesis [19]. Therefore, it is possible that, in $C f b \mathrm{Tg}$ mice, high perilipin expression is induced by PPAR $\gamma$-promoted SREBP-1 activation and subsequent fatty acid synthesis. Furthermore, PPAR $\gamma$ also regulates the expression of CD36 [20], which strongly promotes fatty acid incorporation into adipocytes. In fact, CD36 deficient mice are characterized by smaller adipocytes and decreased adipose tissue weight [21]. Therefore, in our Cfb $\mathrm{Tg}$ mice, increased PPAR $\gamma$ expression might accelerate fatty acid uptake through CD36. aP2 is known to be expressed in other cell types such as macrophages, and it is possible, in our present study, that CfB is also highly expressed in other cell types. CfB overexpression in 3T3-L1 cells showed lipid deposition, and therefore, we speculated that the changes seen in the mice study might have been caused primarily by the changes in adipocytes themselves. In $C f b \mathrm{Tg}$ mice, the effects were more profound in subcutaneous fat than in other fat types. Further study is necessary to fully understand this phenomenon.

Adipocyte specific Cfb Tg mice showed slightly, but significantly increased body weight as well as significantly impaired insulin sensitivity when compared to WT mice. However, these effects were almost completely masked by the western diet-induced obesity (data not shown). We speculated that CfB exerted additive effects on the insulin resistance caused by diet-induced obesity.

$\mathrm{CfD}$, which is downstream of $\mathrm{CfB}$ may act to promote adipocyte differentiation via C3a-C3aR activation [22], was highly expressed in the iWAT of $C f b \mathrm{Tg}$ mice. We also found increased $\mathrm{C} 3$ expression in iWAT of CfB Tg mice. It was suggested that most of C3a, the product of $\mathrm{C} 3$, is converted into acylation-stimulating proteins by serum enzymes, carboxypeptidase $\mathrm{B}$ or $\mathrm{N}$, and helps in promoting lipid storage [23]. Thus, it is also possible that CfB promoted CfD and $\mathrm{C} 3$ expression, adipocyte differentiation and maturation, and lipid accumulation. In conclusion, our findings indicate that $\mathrm{CfB}$ plays a crucial role in late-phase of adipocyte differentiation and subsequent lipid droplet formation.

\section{Funding}

This work was supported by JSPS KAKENHI Grant Numbers
JP16H05555 and JP16K11835.

\section{Conflicting interests}

The authors declare no conflict of interest.

\section{Acknowledgements}

We appreciate the technical assistance from The Research Support Center, Research Center for Human Disease Modeling, Kyushu University Graduate School of Medical Sciences.

\section{Appendix A. Supplementary data}

Supplementary data related to this article can be found at https://doi.org/10.1016/j.bbrc.2017.11.069.

\section{Transparency document}

Transparency document related to this article can be found online at https://doi.org/10.1016/j.bbrc.2017.11.069.

\section{References}

[1] S.P. Weisberg. D. McCann, M. Desai, et al, Obesity is associated with macrophage accumulation in adipose tissue, J. Clin. Invest. 112 (2003) 1796-1808. [2] S.E. Shoelson, J. Lee, A.B. Goldfine, Inflammation and insulin resistance, J. Clin. Invest. 116 (2006) 1793-1801.

[3] V. Tremaroli, F. Bäckhed, Functional interactions between the gut microbiota and host metabolism, Nature 489 (2012) 242-249.

[4] J. Henao-Mejia, E. Elinav, C. Jin, et al., Inflammasome-mediated dysbiosis regulates progression of NAFLD and obesity, Nature 482 (2012) 179-185.

[5] A. Yamashita, Y. Soga, Y. Iwamoto, et al., DNA microarray analyses of genes expressed differentially in 3T3-L1 adipocytes co-cultured with murine macrophage cell line RAW 264.7 in the presence of the toll-like receptor 4 ligand bacterial endotoxin, Int. J. Obese. 32 (2008) 1725-1729.

[6] D. Ricklin, G. Hajishengallis, K. Yang, J.D. Lambris, Complement: a key system for immune surveillance and homeostasis, Nat. Immunol. 11 (2010) 785-797.

[7] M. Matsumoto, W. Fukuda, A. Circolo, et al., Abrogation of the alternative complement pathway by targeted deletion of murine factor B, Proc. Natl. Acad. Sci. U. S. A. 94 (1997) 8720-8725.

[8] G. Engstrom, B. Hedblad, K.F. Eriksson, et al., Complement C3 is a risk factor for the development of diabetes: a population-based cohort study, Diabetes 54 (2005) 570-575.

[9] Z. Liu, Q. Tang, J. Wen, et al., Elevated serum complement factors 3 and 4 are strong inflammatory markers of the metabolic syndrome development: a longitudinal cohort study, Sci. Rep. 18713 (2016), https://doi.org/10.1038/ srep18713.

[10] N. Wlazlo, M.M. van Greevenbroek, I. Ferreira, et al., Low-grade inflammation and insulin resistance independently explain substantial parts of the association between body fat and serum C3: the CODAM study, Metabolism 61 (2012) 1787-1796.

[11] J.M. Moreno-Navarrete, R. Martínez-Barricarte, V. Catalán, et al., Complement factor $\mathrm{H}$ is expressed in adipose tissue in association with insulin resistance, Diabetes 59 (2010) 200-209.

[12] P. Akerblad, U. Lind, D. Liberg, et al., Early B-Cell factor $(\mathrm{O} / \mathrm{E}-1)$ is a promoter of adipogenesis and involved in control of genes important for terminal adipocyte differentiation, Mol. Cell Biol. 22 (2002) 8015-8025.

[13] A. Yamashita, Y. Soga, Y. Iwamoto, et al., Macrophage-adipocyte interaction: marked interleukin-6 production by lipopolysaccharide, Obes. (Silver Spring) 15 (2007) 2549-2552.

[14] J.Y. Huang, M.T. Chiang, L.Y. Chau, Adipose overexpression of heme Oxygenase-1 does not protect against high fat diet-induced insulin resistance in mice, PLoS One 8 (2013) e55369.

[15] S. Gesta, O. Bezy, M.A. Mori, et al., Mesodermal developmental gene Tbx15 impairs adipocyte differentiation and mitochondrial respiration, Proc. Natl Acad. Sci. U. S. A. 108 (2011) 2771-2776.

[16] K. Take, H. Waki, W. Sun, et al., CDK5 regulatory subunit-associated protein 1like 1 negatively regulates adipocyte differentiation through activation of wnt signaling pathway, Sci. Rep. 7326 (2017), https://doi.org/10.1038/s41598017-06469-5.

[17] N. Arimura, T. Horiba, M. Imagawa, et al., The peroxisome proliferatoractivated receptor $\gamma$ regulates expression of the perilipin gene in adipocyte, J. Biol. Chem. 279 (2004) 10070-10076.

[18] D.L. Brasaemle, V. Subramanian, A. Garcia, et al., Perilipin A and the control of triacylglycerol metabolism, Mol. Cell Biochem. 326 (2009) 15-21.

[19] Y. Takahashi, A. Shinoda, N. Furuya, et al., Perilipin-mediated lipid droplet 
formation in adipocytes promotes sterol regulatory element-binding protein1 processing and triacylglyceride accumulation, PLoS One 8 (2013) e64605.

[20] L. Nagy, P. Tontonoz, J.G. Alvarez, et al., Oxidized LDL regulates macrophage gene expression through ligand activation of PPAR $\gamma$, Cell 93 (1998) 229-240.

[21] V. Christiaens, M. Van Hul, H.R. Lijnen, I. Scroyen, CD36 promotes adipocyte differentiation and adipogenesis, Biochim. Biophys. Acta 1820 (2012) 949-956.
[22] N.J. Song, S. Kim, B.H. Jang, et al., Small molecule-induced complement factor D (adipsin) promotes lipid accumulation and adipocyte differentiation, PLoS One 11 (2016) e0162228.

[23] J. Phieler, R. Garcia-Martin, J.D. Lambris, T. Chavakis, The role of the complement system in metabolic organs and metabolic diseases, Semin. Immunol. 25 (2013) 47-53. 